\title{
Tumor necrosis factor inhibitors in the treatment of psoriatic arthritis: a view on effectiveness, clinical practice and toxicity
}

\begin{abstract}
Introduction: Psoriatic arthritis is a common and often severe chronic joint disorder associated with the skin disease psoriasis. Treatment options for psoriatic arthritis patients have considerable changed over the last decade with the wide-spread use of biological therapies, in particular tumor necrosis factor inhibitors. Current clinical experience based on large registries and careful observations now allows to understand the true value of these interventions in daily clinical practice.
\end{abstract}

Areas covered: Literature searches were performed targeting effectiveness, drug survival, toxicity and safety of biological therapies as well as treatment strategies specifically focused on patients with psoriatic arthritis.

Expert opinion: Tumor necrosis factor inhibition is a powerful and effective option for the treatment of severe psoriatic arthritis. The different available drugs have good survival rates and show an excellent balance between effectiveness and toxicity. Switching of inhibitor is feasible but treatment changes should be carefully considered. Novel biological therapies are introduced into the market and will further provide better perspectives for the patient. New questions are also emerging: how to handle long-term remission, can biological therapies be successfully stopped and are co-morbidities sufficiently managed. These questions should be addressed for optimal long-term management of a severe chronic disease.

Keywords: psoriatic arthritis - psoriasis - arthritis - anti-TFN - infliximab etanercept - golimumab - adalimumab - certolizumab 


\section{Psoriatic arthritis, a part of the psoriatic disease spectrum}

Psoriatic arthritis (PsA) is a common chronic inflammatory joint disease that is part of the psoriatic disease spectrum, together with the skin disorder psoriasis $[1,2]$, and that is also frequently included in the spondyloarthritis (SpA) concept $[3,4]$. Psoriasis is a widespread and often severe skin disease typically presenting as psoriasis vulgaris or plaque psoriasis, characterized by hyperkeratosis leading to scaling erythematous plaques most often found on the trunk, the extensor surfaces of the limbs and the head [5-7]. Other clinical presentations such as the guttata form, palmoplantar pustulosis and inverted forms are also well described. At the nails, onycholysis, hyperkeratosis and pitting are frequently recognized. A relatively large number of patients with skin disease, in particular those with psoriasis vulgaris also develop musculoskeletal problems, most commonly arthritis and enthesitis but also spondylitis [8, 9]. Epidemiological data apparently fail to clearly define the relationship between prevalence of skin and joint disease with figures ranging from 0.4 to over $40 \%$ of patients with skin disease also showing signs of arthritis $[9,10]$. The onset of arthritis can occur before, at the same moment or most commonly after the skin symptoms. In addition, some patients do not have skin disease but present with the typical clinical presentation in the joints and a family history of skin disease. These features have been incorporated in the most recent CASPAR classification criteria for PsA [11].

The SpA concept groups a number of different diagnostic entities that share clinical, radiographic and genetic characteristics [3]. Two main subgroups have been defined: axial spondyloarthritis in which the predominant clinical feature is involvement of the spine or sacroiliac joints, and peripheral spondyloarthritis, with arthritis in the limbs being a dominant feature [12]. AxSpA includes ankylosing 
spondylitis, pre-radiographic AxSpA as well as some forms of PsA with spondylitis the main clinical feature. Most patients with PsA will be grouped into the peripheral SpA group, together with inflammatory bowel disease associated SpA, most forms of reactive arthritis and undifferentiated SpA. Upon their detailed description of disease Moll and Wright in addition suggested different subtypes of PsA: oligo-articular, poly-articular, spondylitis, distal interphalangeal joints arthritis, typically associated with nail disease and the most severe form arthritis mutilans [13]. These complex disease definitions and clustering highlight the diversity in clinical presentation of patients with PsA and provide a potential explanation why clinical trials have been relative uncommon in the patients.

PsA is a potentially severe disease as highlighted by the striking numbers of patients with joint damage within 5 years of disease onset, resulting in loss of function and disability as well as increased mortality associated with the disease $[9,14,15]$. Joint inflammation can result in damage and tissue remodeling, features that are typically recognized on X-ray imaging. In PsA both joint destruction with loss of cartilage and the development bone erosions as well as remodeling with juxtaarticular bone apposition leading to the formation of enthesophytes and eventually joint ankylosis occur $[16,17]$. Both features contribute to progressive loss of function. A systemic effect of the chronic disease process with clear evidence for increased cardiovascular morbidity in this patient group has also been demonstrated $[18,19]$ [20-22]. Adding the additional dimensions of psychiatric disorders such as depression, alcohol abuse, and metabolic disorders such as type II diabetes and obesity, all associated with the skin disease and at least to some extent with joint disease [23, 24], concepts and strategies should ideally aim for a holistic approach towards psoriatic disease. 


\section{The challenge of treating patients with psoriatic arthritis}

Despite its prevalence and severity, treatment options for PsA patients remained limited and the number of clinical trials with a specific focus on PsA astonishingly low. Current approaches support the use of non-steroidal anti-inflammatory drugs in mild cases and the use of immune modulating drugs, typically referred to as disease modifying anti-rheumatic drugs (DMARDs), in more severe cases [25-28]. In this context, common drugs are methotrexate (MTX), leflunomide and sulfasalazine. Although MTX has been used for decades in PsA treatment, clinical trial evidence is limited and its use is mainly based on experience and expert opinion [29]. The effect of leflunomide, another immune modulator, has been better studied in at least one randomized controlled trial including 190 patients [30] but in most countries, it is considered a second-line treatment. Sulfasalazine was also more extensive studied in different small trials and also appeared effective [26]. Cyclosporine probably had stronger effects, mostly on the skin but its toxicity limit its use for PsA $[25,28]$. However, a large number of patients do not respond in a satisfactory way. Biological therapies, in particular targeted therapies against cytokine tumor necrosis factor alpha are then a preferred choice $[25,27,28]$.

TNF is increased in both skin and joint of PsO and PsA patients [6] and its specific targeting has been highly successful in clinical trials. Different TNF inhibitory strategies have been studied in randomized controlled trials and are now available. Infliximab (IFX) is a chimeric mouse-human antibody administered every 6 to 8 weeks by IV infusion [31-35], adalimumab is a fully human antibody administered every two weeks by SC injection [36-40], alternative antibodies include human antibody golimumab [41-44] and certolizumab, a PEG-linked antibody [45]. 
As an alternative to blocking antibodies, etanercept (ETN), a soluble receptor administered SC once or twice a week is also available [46-48]. The efficacy of these anti-TNF drugs has been well studied in phase II and III clinical trials, further analyzed in extension studies and confirmed by meta-analysis [26, 49, 50]. These analyses did not suggest major differences in efficacy or toxicity between the different anti-TNF strategies used.

Recently new drugs have been explored for the treatment of PsA. Apremilast is a phosphodiesterase antagonist with good effects on PsA in phase II and III trials [51, 52]. Ustekinumab (antiIL12/23) is an antibody against the common p40 unit of the cytokines IL12 and IL23 with strong effects on the skin disease and has also been studied in PsA [53-56]. Both drugs have now been approved for the treatment of PsA by regulatory agencies. Phase II trial using brodalumab, an antibody directed against the IL17A receptor, and secukinumab, an antibody directed against IL17A, showed a good effect in PsA patients $[57,58]$. Another molecule of interest is abatacept, a modulator of $\mathrm{T}$ cell costimulation [59].

The introduction of these biological therapies has dramatically changed the perspective of patients with PsA. Anti TNF strategies (TNFi) are currently and increasingly used in a large number of patients. In this summary, we look specifically at the effectiveness of TNFi in PsA treatment beyond the stage of the clinical trials. From this perspective we discuss current data on drug survival, effectiveness and side effects of these drugs as insights have evolved in over a decade of their use. In the last section, we provide our personal view on the use and usefulness of these drugs as cornerstones in the treatment of PsA. Data on other biological therapies, in particular ustekinumab outside of clinical trials, are not yet available. 


\section{Effectiveness of TNFi in psoriatic arthritis: drug survival}

Randomized controlled clinical trials are essential to demonstrate the efficacy of drugs in a specific patient population compared to a control group. In arthritis trials, outcome measure typically reflect an improvement in the number of painfull and swollen joints or a quantitative index incorporating such physician determined outcome measurements as well as patient reported outcomes. The real-life position and use of a drug is more complex: efficacy, patient satisfaction, balance between side-effects and benefits, short and long-term toxicity and availability of altnerative strategies are just of number of features that come into play. Patients in daily practice are also different from those that fulfill trial inclusion criteria. Therefore an assessment of treatment effectiveness can be based on drug survival in prospective cohorts as well as patient-reported outcomes. For TNFi in the treatment of PsA, such data have become available over the last years and allow us to better position the impact of TNFi in these patients.

The Norwegian DMARD (NOR-DMARD) registry prospectively includes all patients with chronic arthritis from five different Norwegian centers who started a disease modifying regimen (classic or TNFi) [60]. The enrollment success is estimated at $85 \%$. One-year drug survival for TNFi was recorded for RA, PsA and AS patients. The reported data set included 1,019 patients of whom 150 patients had PsA [60]. Taking into account the use of a second TNF inhibitor, 172 treatment regimens in PsA patients were assessed. Patient characteristics are summarized in Table 1. Crude drug survival was assessed by Kaplan-Meier analyses and log-rank tests. Crude survival at one-year in PsA patients was $77.3 \%$ and significantly better than in RA patients. Cox proportional hazards regression adjusting for age, sex, disease activity 
and use of MTX and checked for proportionality identified a hazard ratio (HR) of PsA vs. RA of 0.76 (95\%CI: $0.53-1.07)$ corresponding with a p-value of 0.12 . In the whole group, MTX use was associated with much lowered risk of treatment termination (HR $0.53(95 \% \mathrm{CI}: 0.43-0.65)-\mathrm{p}<0.001)$, an effect reported to be consistent over diagnoses and different TNF inhibitors. Effectively, Kaplan-Meier analysis in PsA patients alone, was significantly different between non-MTX and MTX users favoring drug survival in the latter group. When entered as co-variates in the model, no significant differences was found between IFX, ETN and ADA when only TNFi naïve patients were taken into account. Inclusion of all patients suggested a lower risk of treatment termination with ETN as compared to IFX and ADA but the authors pointed towards the limitation that ETN was more often the first-choice TNFi compared to the other two.

The reasons for discontinuation in PsA patients were lack of efficacy (17.9\%) and adverse events (69.2\%), patient's request and other or unknown (combined 12.8\%). Of note, no patient receiving combination of TNFi and MTX discontinued TNFi within the one-year time window of the analyses.

These interesting observations with regards to concomitant MTX treatment stimulated the NOR-DMARD investigators in revisiting this issue in a larger group of patients a number of years later [61]. 440 patients with PsA were selected from the registry if they were receiving their first TNFi and separated into two groups, monotherapy or combination with MTX. A small number of patients using other DMARDs in combination with TNFi were not considered. The primary analysis of effectiveness was based on non-joint score dependent outcomes as about $30 \%$ of the patients did not have at least one swollen joint at baseline. 330 patients with swollen joint(s) at baseline were included in a secondary analysis setup. Of note, $70.6 \%$ of the patients in 
the mono-therapy group had previously used MTX. In the first analysis, patients not receiving MTX performed worse at month 3 on the Physician Global Score, a difference that was no longer found at month 6 and 12. Similar observations were made in the secondary analysis cohort. With regards to drug survival, Kaplan Meier analyses showed a significant difference favoring concomitant MTX at year 1 and year 2 but no longer at year 3 (with $\mathrm{p}=0.07$ suggesting a persistence of the trend). When separated by the three main TNFi used in these patients, a 3-year statistically significant difference was found in patients using IFX, and a trend was suggested in the ADA group. In contrast ETN curves for both groups were almost overlapping. The IFX without co-medication has significantly increased discontinuation ratios due to adverse events. Multivariate analysis identified the use of concomitant MTX $(p=0.04(95 \%$ CI:0.52-0.99)) and current smoker status $(p=0.02$ (95\% CI:1.08-1.13)) as predictors of drug survival.

The DANBIO registry prospectively includes patient receiving biological treatment from 25 rheumatology departments in Denmark and covers about $90 \%$ of the patients treated with a biological drug in routine clinical care. Drug effectiveness and survival were studied in 764 PsA patients [62]. Data acquisition for this study started in 2000 and database lock was done in 2009. Patient characteristics are summarized in table 1. Crude drug survival was analyzed by Kaplan-Meier analyses and log-rank tests. Cox regression analyses were used for detailed analysis of factors associated with drug survival. Drug survival rates at year 1 and 2 were $70 \%$ and $57 \%$ respectively. No differences were found between patients receiving IFX, ADA and ETN. Cox regression identified female sex (HR 1.42 (95\%CI: 1.11-1.80) - p-value 0.005), high VAS global score (HR 1.10 (95\%CI: 1.04-1.17) - p-value 0.001), low CRP at baseline (HR 1.4 (95\%CI: 1.09-1.78) - p-value 0.008) and absence of MTX 
use (HR 1.37 (95\%CI: 1.07-1.75) - p-value 0.013) as risk factors for discontinuation. Of interest, in this study type of biological drug was not significant.

Reasons for discontinuation were analyzed in detail. Lack of efficacy was responsible for $52 \%$ of the withdrawals and adverse effect for $28 \%$. A stratified regression analysis including only adverse events as reason for discontinuation identified female sex but also the use of IFX and lack of concomitant MTX use as risk factors. HR of IFX vs ADA or ETN were 0.49 and 0.46 respectively. These factors were not significant when stratification was based on lack of efficacy.

The DANBIO and a similar registry from Iceland (ICEBIO) that started prospectively in 2007 with retrospective registration of biological treatments started before that date, was also used to study the impact of different IFX dose regiments on response and drug survival, specifically in patients with PsA [63]. The ICEBIO registry covers over $95 \%$ of all biological treatment given to patients with rheumatological disorders in Iceland. 462 patients were studied (376 DANBIO - 86 ICEBIO). Characteristics of the patient populations can be found in Table 1. Median starting dose $3.1 \mathrm{mg} / \mathrm{kg}$ in Denmark was significantly higher than in Iceland $(2.3$ $\mathrm{mg} / \mathrm{kg}$ ), which can be explained by specific treatment guidelines in Iceland. At 1 year $58 \%$ (Denmark) and 66\% (Iceland) of patients were still on the drug with drug survival significantly different between the countries. This was not explained by starting dose. As before drug survival was shorter in patients not receiving concomitant MTX in Danish patients $(\mathrm{p}=0.009)$ and a similar trend was seen in Iceland $(\mathrm{p}=0.1)$. Interestingly, start of treatment in later years was also associated with lower drug survival. This can be explained to some extent by the availability of treatment alternatives. $25 \%$ of the patients stopped the drug because of lack of efficacy whereas $29 \%$ stopped due to adverse effects. 
The British Society for Rheumatology Biologics Register (BSRBR) recruits patients with chronic arthritis treated with biological therapies in the UK. The registry also includes patients with PsA. Data on drug survival were first reported for patients that started TNFi between 2002 and 2006 [64]. Patient characteristics are shown in Table 1. Kaplan-Meier and Cox proportional hazard regression analyses were applied to the data. Drug survival data were available for 566 PsA patients: 316 treated with ETN, 162 with IFX and 88 with ADA as first TNFi. $75 \%$ of patients remained on their first TNFi in the first year, with $9.5 \%$ discontinuing due to lack of efficacy and $10 \%$ due to adverse events. Survivor functions over 3 years suggested a tendency towards shorter persistence of treatment with IFX, compared to the two other drugs. Infusion reactions were the most common cause of drug discontinuation. HR analysis indicated that female sex (HR:1.3 95\%CI:1.0-1.7), base-line comorbidity (HR:1.5 95\%CI:1.1-2), and IFX rather than ETN (HR:2.8 95\%CI:2.1-3.7) were associated with worse drug survival. Further analysis focused on discontinuation due to inefficacy, multivariate analysis only found IFX rather than ETN associated (HR 3.8 95\% CI:2.0-7.3) to be significant. In line with the other studies mentioned here, concomitant DMARD therapy and higher baseline disease activity showed better survival. In this register also patients using a second TNFi were analyzed [64]. Follow-up times were shorter and the number of patients (178) limits the conclusions that can be drawn but survival function was 0.74 at 12 months, a value lower than 0.82 recorded for all patients receiving a first TNFi course at year 1.

The BIOBADASER registry is a Spanish drug registry that was established in 2000 and includes patients with rheumatic disease that are being treated with biological therapies [65]. Over 100 practices (both private and hospital based) participate in the effort. Coverage is estimated at about $60 \%$ of patients treated with 
these therapies in Spain. Carmona et al. presented a first analysis of drug survival in 2006 [65]. The team compared spondyloarthritis, including PsA, as a group with rheumatoid arthritis but also paid attention to the individual subtypes of SpA. 1,524 patients with SpA were included at that time point, of which 570 were diagnosed with PsA. The number of RA patients in the registry was 4,006 . At the time of the report ADA use in SpA, in contrast to IFX and ETN was very low (less than 1\%). PsA patient characteristics are shown in Table 1. Survival of anti-TNF was significantly greater in the SpA group compared to the RA group at year 1, 2 and 3. Drug survival was $0.88,0.81$ and 0.73 for the PsA patients at year 1,2 and 3 respectively. For RA patients this was $0.83,0.72$ and 0.65 . HR for discontinuation in the $\mathrm{SpA}$ group as compared to RA was $0.66(95 \% \mathrm{CI}: 0.57-0.76)$ after correction for age, sex and infliximab use. This was observed for both IFX and ETN treatment. Adverse events accounted for $45 \%$ of the discontinuations in the SpA group and lack of efficacy for 34.6\%. Within the SpA group the HR for AE were not different. Further analyses demonstrated that the HR for an $\mathrm{AE}$ in the $\mathrm{SpA}$ group compared to RA was 0.80 (95\%CI: 0.7-0.91) after correction for disease duration, age and use of IFX. However, the HR for discontinuation of PsA compared to RA was 0.81 with $95 \%$ CI $0.66-0.99$. This effect lost its significance after correction for age.

This was further assessed in a follow-up study using the BIOBADASER 2.0 database [66]. Here, the authors demonstrated that patient younger than 65 more frequently discontinue a TNFi due to inefficacy whereas patients over 65 are more likely to stop treatment due to adverse effects. They further found that RA patients were more likely than SpA patients to discontinue treatment with TNFi due to either inefficacy or adverse events. 
A similar study was undertaken by the South Swedish Arthritis Treatment Group (SSATB) who followed up PsA patients since 1999 [67]. 261 PsA patient starting a TNFi were included and prospectively followed. Drug survival was analyzed using Kaplan-Meier and log-rank test and by Cox proportional hazards regression analyses. Patient characteristics are shown in Table 1.29 patients received ADA, 119 ETN and 86 IFX. 161 patients received concomitant MTX at treatment initiation. Of these, 16 stopped MTX treatment during the observation period. No statistically significant differences were found between these two groups. Patients treated with MTX showed a trend for increased drug survival $(p=0.10)$. In the smaller group of patients who withdrew treatment because of adverse event, this protective effect of concomitant MTX therapy was more outspoken $(\mathrm{p}<0.01)$. Survival rates were not specifically reported at year 1 or 2 . The multivariate analysis confirmed that the use of MTX, baseline higher CRP and ETN compared to IFX had significant effects on drug survival. In further analyses both the MTX and ETN effect were linked to significantly fewer adverse events and not to treatment failure.

A single center retrospective study by Duclos et al. including 770 patients with chronic arthritis treated with TNFi only separated the RA and SpA groups [68]. Within the latter 60 patients were diagnosed with PsA. They confirmed the observation that drug survival is longer in SpA patients than RA patients (HR 1.6 O5\%CI: $1.20-2.13-p=0.001)$ but do not provide specific data for PsA patients.

\section{Is it useful to switch a TNF inhibitor in case of loss of effect or adverse events?}

The data summarized above demonstrate that in a substantial number of patients with PsA, a first TNFi may not be sufficient to control disease activity, either due to non-responsiveness or loss of response, or that patients may be required to cease 
therapy due to adverse events. As only few strategies to treat PsA are available, it is necessary to understand whether a second or even a third TNFi can be used safefully and successfully in this group of patients. Answers towards these questions should be specifically sought in the PsA patients and not extrapolated from what we know from other diseases such as rheumatoid arthritis as disease mechanisms but also reasons for switching are likely different.

A first analysis was presented from the BIODASER registry reporting on 15 patients with PsA within a larger cohort of 488 patients that switched treatment [69]. Odd's ratios for survival of the second TNFi appeared similar between these PsA patients (0.81; 95\%CI:0.65-0.90) and those with RA (0.79 95\%CI:0.74-0.83). However, the number of RA patients (194) largely outnumbered that of patients with PsA. Another small group of 15 PsA patients was presented by Conti et al. suggesting that failure of the initial TNFi did not exclude a good response towards a second drug [70]. Haberhauer et al. reported on 21 PsA patient that received a second and 3 PsA patients a third TNFi [71]. The analysis suggested that patients with PsA showed a better response towards a second TNFi than patients with rheumatoid arthritis or ankylosing spondylitis with an adequate response in $76 \%$ of the PsA patients compared to $33 \%$ and $46 \%$ in the other groups respectively. Again, the number of patients studied limits the potential conclusions that can be drawn but these data at least indicated that a second TNFi may be useful.

More extensive data were recently reported from the DANBIO registry [72]. $548(33 \%)$ of 1422 PsA patients that started a TNFi switched to a second TNFi during a decade of follow up (but with median follow-up 2.3 years). Of note, 632 patients were still on their original TNFi and 242 had stopped treatment without starting a second TNFi. $57 \%$ of switchers did so for lack of effect and $28 \%$ for adverse events. 
Of this group, 245 continued their treatment with the second TNFi at database lock but 189 (34\%) had switched to a third TNFi and 114 stopped the TNFi. Lack of effect was again the main reasons for switching (62\%) and 43/189 switched to a third course for adverse events. 57 out of 189 switched effectively to a fourth course and 20 out of these even to a fifth TNFi. Epidemiological data suggested that switchers were more frequently women, with shorter disease duration and higher HAQ, DAS28, fatigue and pain scores as well as more swollen and painful joints. Baseline disease activity was not different between switchers and non-switchers. Drug survival decreased in switchers from 2.2 years (first course) towards 0.7 years (second course), but disease activity was significantly reduced after first, second and third course. However, response rates were significantly lower after the second and third course compared to the first. In a multivariate regression analysis only lower fatigue score was a predictor of survival with the second TNFi.

A smaller sample of 95 PsA patients that switched TNFi were reported from the NOR-DMARD registry and compared to non-switchers (344 patients) [73]. This group included all patients that started a first TNFi and did not use a second TNFi. The switchers had significantly poorer responses towards the second TNFi compared to the response of the non-switchers to the first. Baseline data showed that the switch group had a lower CRP and swollen joint count before starting their second TNFi, suggesting an effect of the previous intervention. Nevertheless the switchers had a stronger response with their second TNFi as compared to the last reponse towards their first TNFi. At 3 months no specific differences were found between the switchers that discontinued their first TNFi due to adverse events $(n=35)$ or those that discontinued due to lack of resonse $(n=49)$. Drug survival of the second TNFi was significantly poorer than that observed in the non-switchers $(0.74$ for first TNFi (all); 
0.83 for non-switchers and 0.56 for second TNFi). Nevertheless, there was no drug survival difference between switchers that used a second TNFi because of adverse effects or lack of response.

In a subanalysis of the BSRBR registry 178 patients with PsA were found to have received a second TNFi [64]. At 12 months drug survival was 74\% with survival function slightly lower than for the first course 0.74 (95\% CI $0.71-0.78)$ vs. 0.82 (95\% CI 0.79-0.85). An interesting short report was based on a survey in the north-west of England, which noted that $17 \%$ (94) of 548 patients treated with a TNFi had switched over time with $3 \%$ switching between 3 or 4 biological therapies [74]. About $60 \%$ of these patients had an adequate response to the second TNFi.

\section{Can we assess the true benefit of TNF inhibitors for PsA patients?}

The registry data also provided important insights into the true effectiveness of the treatment in the perception of the patients. 596 patients with PsA included in the BSRBR were specifically studied to assess the risk-benefit profile [75]. Most data were reported with the time-window defined at 6 months. Unfortunately, the authors were not able to specifically include a PsA-DMARD only patient group as controls but had to compare their PsA biological therapies-treated patients with a rheumatoid factor-negative rheumatoid arthritis patient cohort to compare side effects. Treatment response was based on assessment of the DAS28 score. This calculated score includes an assessment of swollen and tender joints, CRP/ESR and patient global assessment. EULAR criteria were used to define good, moderate and no response. At 6 months, $37.5 \%$ of PsA patient reached a good response and another $38.3 \%$ a moderate response, an effect sustained at 12 and 18 months. At month $1868.2 \%$ of the PsA patient could be considered responders. There were no notable differences in 
responses between the three agents used by the patients in this cohort (IFX, ETN, ADA), or between patients using cocomitant MTX or not. Remission as defined by EULAR criteria was achieved in 131 patients (35.2\%) at 19 months. Multivariate analysis suggested that age, female gender and the use of steroids resulted in lower response rates to TNFi. This was confirmed in the analysis for remission again identifying a lower chance to achieve remission for older and female patients.

From patient and physician perspective monitoring of side-effects is obviously important. Analysis of TNFi associated side-effects in this setup included 1776 person years of follow up [75]. No differences were found between the TNFi treated PsA patients and the control RF-RA patient group. Of note there were 14 malignancies in the TNFi group but no lymphomas. Overall incidence, patient group size and time of follow-up were too small to detect any important difference in malignancy if it existed.

Evaluation of effectiveness in the NOR-DMARD registry was focused on Health related quality of life assessment based on the SF-36 questionnaire [60]. This approach may be closer to the daily life of the patient than the DAS28 score mentioned above. Baseline adjusted changes in all 8 SF dimensions were superior in PsA and AS patients as compared to RA patients. Of these, the mental and social in contrast to the physical dimensions did not get statistical signficance. The follow-up study in the NOR-DMARD registry that focused on cocomitant use of MTX, did not show significant differences in changes from baseline among the groups at 6 and 12 months [61].

In the DAN-BIO registry, outcome was assessed with different instruments: health assessment questionnaire (HAQ), DAS28 score as well as VAS scores for pain, patient global and fatigue, CRP and joint counts [62]. All of these outcome 
parameters significantly improved compared to baseline up to 5 years follow-up. With also CRP as well as tender and swollen joint counts available, the investigators were able to calculate EULAR responses as well as ACR20, 50 and 70 response criteria. Complete data were available for 63 and $56 \%$ of the patients respectively. $54 \%$ of the patients with data available obtained a good EULAR response during the first 6 months of treatment, whereas $27 \%$ achieved a moderate response with $19 \%$ having no response. These numbers were in line with ACR response criteria: ACR20 in 59\% of the patients, ACR50 in 45\% of the patients and ACR70 in 24\%. Multivariate analysis identified younger age, high CRP and male sex as predictor of good response. For ACR20 MTX use, high VAS global health and high CRP were associated with response. In a similar analysis for ACR50 response, only CRP $>10 \mathrm{mg} / 1$ was associated. These numbers were translated into interesting number of treat figures predicting that only 1 in 7 patients achieved ACR70 if the CRP level was normal, but that 1 in 3 did with CRP levels higher than $10 \mathrm{mg} / \mathrm{ml}$.

The SSATG group also reported on outcome [67]. Principal parameters were the EULAR response criteria based on 28 joint counts (not taking int account PsA patients with spondylitis only) as well as 50\% improvement in VAS global and VAS pain. Data are reported for up to 12 months. Good EULAR response was found in $55 \%$ and moderate EULAR response in $75 \%$ of the PsA patients. $50 \%$ improvement in VAS global and VAS pain was found in about $50 \%$ of patients. There were no differences between MTX treated patients and those without, or between type of antiTNF therapy or clinical presentation. The LUNDEX (Lund efficacy index) is a measure to calculate the fraction of patients that fulfilled reponse criteria and remained on treatment. Here there was a trend for higher LUNDEX values in patients treated with concomitant MTX. 


\section{Toxicity and anti-drug antibodies}

Overall, the data from the different clinical trials as well as from the extended registries as summarized above, do not indicate specific toxicity associated with PsA as compared to other diseases for which TNFi are used. Most side-effects are related to injection site reactions, infections and infusion reactions in case of IFX treatment. Of note, some studies appear to underline the safety of biological treatment in PsA patients with latent tuberculosis and hepatitis $\mathrm{C}$ virus infection $[76,77]$. With regards to malignancy, up till now no specific signal of increased risk in PsA patients is consistently detected. Some caution should however be in place [78] and long-term effects carefully monitored. In particular concerns about melanoma may be warranted as PsA patients, in particular those with severe skin disease may have had extensive exposure to UV light in case of phototherapy. Earlier analyses suggested that under treatment with biological therapies non-melanoma skin cancers may more easily develop [79].

Paradoxical effects of TNFi such as exacerbation or new onset of psoriasis have been described in different patient groups. Altogether these unexpected manifestations of disease remain relatively rare and poorly understood. Aspects of their pathophysiology were recently reviewed extensively [80]. From the clinical practice point of view, a careful diagnostic approach seems warranted and expert dermatologist opinion required to exclude other skin disorders mimiching clinical features of psoriasis.

Poor long-term drug survival and effectiveness have been linked to the formation of anti-drug antibodies in different immune-mediated disorders for which 
TNFi have been used [81]. Antibodies against IFX and ADA appear most common, and have been linked with reduced drug activity in patients with rheumatoid arthritis and inflammatory bowel disease. Only limited data are currently available for PsA patients [82]. It remains unclear whether such antibodies are more prevalent in disorders typically associated with auto-antibody formation such as rheumatoid arthritis as compared to PsA and other forms of SpA.

\section{Expert opinion on the use of TNFi in daily practice for the treatment of PsA}

A little over a decade ago, treatment of PsA was an enormous challenge in a large group of the patients. Although NSAIDs could give a good response in patients with limited disease, there were little options available for patients with severe involvement. Therapy-resistant monoarthritis of large joints, polyarticular disease and severely destructive arthritis mutilans combined with the burden of skin disease were major challenges. Often, patients were treated with multiple DMARDs, including cyclsporin as well as steroids. Even in the absence of solid evidence, these strategies appeared the only options, despite the considerable side-effects. The introduction of TNFi for the treatment of both skin and joint disease in the psoriatic disease spectrum has dramatically changed the treatment options and the prognosis of patients.

Clinical trial data reflect only some aspects of daily clinical practice. The efficacy of the different anti-TNF drugs on both joint and skin manifestations was undisputed in the trial setting and translated well into clinical practice. Data from large registries have been published and discussed above. The registry data also reflect well our personal experience in a tertiary referral center. Drug survival, in some patients over a decade, is good to excellent and patient as well as physician 
satisfaction with the health status of many PsA patients is similar. The drug survival data, when considered as a proxy for effectiveness of the therapy, should nevertheless be considered with caution. Over the decade concerned the number of TNFi available has increased and we are now most likely entering an area of transition in which alternative biological strategies will become available. On one hand, we assume that the increased availability of alternatives, whether TNFi or different drugs, will lower the threshold to switch therapy in case of incomplete clinical response. Such a strategy can be successful, as has become clear from the data of patients that switched their TNFi, but may also carry some risk when defining treatment strategies. PsA, like other chronic immune-mediated diseases, has a variable course and episodes of low and high disease activity may alternate. External factors such as excessive or unusual strain on joint due to occupational or leisure activities may trigger flares of joint disease; psychological stress situations and co-morbidities may have an impact on the patient's disease specific and global health assessment. In this context, we advocate a cautious approach to switching biological therapies. The perceived loss of response during one out-patient visit may be temporary. A clinical approach with temporary adjustments of therapy, e.g. by associating NSAIDs or optimizing pain-killers or imposing rest, and re-evaluation of the patients may prevent the necessity of switching therapies and support survival on the initial drug chosen. As the number of options to treat patients remains limited, drug survival is a priority in the treatment of such a chronic disease.

On the other hand, novel targets and strategies are eagerly awaited. Head to head comparisons in clinical trials are difficult to do and the patients that can be included in clinical trials with new biological therapies may show different characteristics in 2014 as they did a decade ago. The evidence that anti-IL12/23 and 
anti-IL17 strategies are successful in controlling skin and joint symptoms is a boost for the treatment of patients. This is not only of interest for those with a lack or loss of response towards TNFi but also should direct the attention towards the specific effectiveness of different biological therapies to distinct disease manifestations: synovitis as apposed to enthesitis, skin as opposed to joint disease, nail involvement, destructive arthritis etc. We anticipate that the combined data from clinical trials and clinical practice will provide further guidance towards the optimal choice of strategies for the individual patient. Obviously better molecular characterization of the disease processes and of the individual patients will be equally essential to move forwards towards personalized medicine.

Another important real-life issue in the approach towards PsA patients, is what to do after a patient has achieved disease remission. The unpredictable disease course of PsA suggests that such remission may be long-standing. Taking into account the high cost associated with the drugs and the potential long-term side effects, therapyreduction or stop strategies require more attention. In this context, the clinical community should also focus further on potential issues with reintroduction of therapies. This may be a challenge in patients that have developed anti-drug antibodies.

Long-term management of PsA also should take into account co-morbidities $[20](\S$. Some of these may be directly linked to inflammation and insufficient disease control, e.g. the increase in cardiovascular risk. TNFi appear to be effective in ameliorating at least some of the risk factors involved [83]. Other co-morbidities such as malignancy may be linked to the use of therapies, not only biological therapies but also DMARDs like MTX and cyclosporine or phototherapy for skin disease. Depression, alcohol and substance abuse appear linked to overall wellbeing of the 
patient although a direct connection to the disease processes in PsA and psoriasis has not been excluded. The impact of the TNFi and other biological therapies on these comorbidities is far from understood. As suggested above, data and concepts that apply to rheumatoid arthritis patients may not fully apply to PsA patients. Nevertheless, the successful control of signs and symptoms caused by inflammation at least suggest that TNFi will also have a positive effect on cardiovascular complications of disease. To demonstrate such effects, innovative approaches in available cohorts are most likely needed and should be encouraged. The co-morbidity impact of different biological therapies may not be similar. Recently some concerns have risen about cardiovascular impact of ustekinumab, although the eventual negative effect is controversial [84-86].

\section{Declaration of interest}

R.L has received grant support or speaker and consultancy fees from Abbvie, Boehringer-Ingelheim, Celgene, Johnson \& Johnson, Merck, Pfizer and UCB. K.D.V. has received grant support or speaker and consultancy fees from Abbvie, Celgene, Johnson \& Johnson, Merck, Novartis, Pfizer and UCB. 


\section{Article highlights box}

- Psoriatic arthritis is a common chronic inflammatory skeletal disease potentially leading to structural damage, loss of function and disability and should therefore be optimally managed.

- Treatment options in psoriatic arthritis have been limited for a long-time to the use of anti-inflammatory drugs and immune-modulators such as methotrexate, sulfasalazine and more recently leflunomide that were at best moderately effective.

- The introduction of biological therapies, in particular antibodies or soluble receptors directed against cytokine tumor necrosis factor alpha, has dramatically changed the management of patients with psoriatic arthritis.

- Long-term drug survival is often used as a proxy for treatment effectiveness and shows excellent performance of the different tumor necrosis factor inhibitors with a good balance between effects and toxicity.

- Methotrexate use may improve drug survival, in particular in patients that are treated with infliximab, a first generation tumor necrosis factor inhibitor.

- New biological therapies for the treatment of psoriatic arthritis are appearing, e.g targeting interleukin-17 or interleukins $12 / 23$. These options currently look promising and the field is therefore likely to evolve over the next years, most likely to further benefit for the patients. 


\section{Figure legends}

Figure 1: Overview of patient responses in major anti-TNF trials in psoriatic arthritis. The percentage of patients achieving an ACR20 response (20\% reduction in number of swollen and painful joints combined with $20 \%$ in minimum 3 out of 5 other outcome measures (ESR/CRP, physician global score, patient global score, patient pain score and health assessment questionnaire). No head to head comparisons are available. All differences were significant in the clinical trials. Data derived from references $[35,37,44-46]$. 